\title{
Prevalence of metronidazole resistant Helicobacter pylori strains among Chinese peptic ulcer disease patients and normal controls in Hong Kong
}

\author{
C K Ching, K P Leung, R W H Yung, S K Lam, B C Y Wong, K C Lai, C L Lai
}

\begin{abstract}
Background-A study was conducted to evaluate the prevalence of metronidazole resistant Helicobacter pylori strains among the Chinese in Hong Kong. The efficacy of the triple therapy that contains metronidazole as one of the anti-microbial agents in eradication of the metronidazole susceptible and the metronidazole resistant strains was also assessed.
\end{abstract}

Methods-Culture for $H$ pylori was attempted from antral biopsy specimens of 70 peptic ulcer and 51 control subjects. Successfully cultured $H$ pylori strains were tested for metronidazole susceptibility. Twenty six peptic ulcer disease subjects who had received a course of triple therapy were also reassessed four to six weeks later for successful eradication of $\mathrm{H}$ pylori infection.

Results and conclusions-H pylori was successfully cultured from antral biopsy specimens in 69 of $80(86 \%)$ of the infected subjects. The overall metronidazole resistance rate was $53.5 \%$ ( 37 of 69 ). There was a significantly higher metronidazole resistance rate among $H$ pylori isolates from the asymptomatic controls ( 20 of 25 ) than the peptic ulcer disease subjects (17 of 44) $(p=0.0007)$. Twenty three of 32 $(73 \%)$ women and 14 of $37(38 \%)$ men harboured the metronidazole resistant strains. There was no sex or age difference as far as the prevalence of metronidazole resistant strains were concerned within each study group. Pre-treatment metronidazole susceptible $H$ pylori were significantly more likely to respond to the triple therapy used than those with the metronidazole resistant ones (14 of $15 v$ five of 10) $(\mathbf{p}=\mathbf{0 . 0 2 1})$.

(Gut 1996; 38: 675-678)

Keywords: Helicobacter pylori, metronidazole susceptibility, Chinese.

Treatment of peptic ulcer disease has been revolutionised ever since the demonstration of significantly lower ulcer, both duodenal and gastric ulcer, relapse rates after successful Helicobacter pylori eradication. ${ }^{1-6}$ This led to the recent recommendation by the National Institute of Health Panel that anti- $H$ pylori therapy should be considered for every patient with peptic ulcer disease when it is associated with $H$ pylori infection. ${ }^{7}$ Such a policy is more or less adopted by most gastroenterologists worldwide. The most widely used combination therapies currently consist of either a proton pump inhibitor or bismuth compound together with two antibiotics. One of the antibiotics used is a nitroimidazole, for example, metronidazole. Previous reports showed that the overall $H$ pylori eradication rate depended significantly on the organism's susceptibility towards metronidazole, ${ }^{8-12}$ with a significantly lower eradication rate in those with the metronidazole resistant strains than those with the metronidazole susceptible ones. It has been demonstrated in a recent survey by Glupczynski et al ${ }^{13}$ that natural resistance of $H$ pylori against nitroimidazoles occurs between $7 \%$ (Madrid) to $49 \%$ (Athens) in Europe. In contrast, it is approximately $80 \%$ in the developing countries for example in Africa. ${ }^{1415} \mathrm{Little}$, however, is known about the prevalence of metronidazole resistant $H$ pylori strains among the Chinese in Hong Kong. We have therefore conducted a study among peptic ulcer disease patients and a group of healthy volunteers to report in the prevalence of metronidazole resistant $H$ pylori strains and to assess the influence of pre-treatment metronidazole resistance on the final eradication rate by combination therapy that includes metronidazole as one of the antimicrobial agents.

\section{Methods}

\section{Subjects}

Seventy patients with peptic ulcer disease were entered into this study. Twenty six (25 duodenal ulcer and one gastric ulcer) cases were given anti- $H$ pylori therapy (16 males, mean age $47 \cdot 8$ years; 10 females, mean age 52.7 years), and the other 44 (38 duodenal ulcer and six gastric ulcer) cases ( 28 males, mean $44 \cdot 7$ years; 16 females, mean age 56.4 years) received either omeprazole or ranitidine as anti-ulcer therapy. Fifty one healthy volunteers $(23$ males, mean age 48.1 years; 28 females, mean age 60.6 years) recruited from medical exhibitions were included as controls in this study. The 26 peptic ulcer disease patients received omeprazole 20 mg twice daily (for six weeks) and metronidazole $300 \mathrm{mg}$ four times daily (for 14 days) in combination with either clarithromycin $250 \mathrm{mg}$ four times daily (13 cases) or amoxycillin 500 mg four times daily (13 cases) (for the first 14 days). Repeat endoscopy and gastric biopsies was performed four to six weeks on 25 of these 
26 patients (one defaulted from follow up) after completion of anti- $H$ pylori treatment to reassess the eradication rate by urea broth test and histological examination.

\section{Endoscopy and biopsy specimens}

All the above patients underwent upper gastrointestinal endoscopy after local lignocaine throat spray. Six biopsy specimens were taken from the antrum, two for culture and sensitivity testing, two for urea broth test, ${ }^{16}$ and two for histological examination ${ }^{17}$ as described before. Only two specimens were taken from the corpus of the stomach for histological examination.

\section{Diagnosis of $\mathrm{H}$ pylori infection}

A positive diagnosis of $H$ pylori was made when at least two of the three tests, the urea broth test, histology, or microbiology were positive. Eradication of $H$ pylori after triple therapy was confirmed by a negative urea broth test and histology showing absence of $H$ pylori both in the antrum and in the corpus.

$\mathrm{H}$ pylori culture and sensitivity

Antral biopsy specimens obtained from these patients were totally embedded into Stuart transport medium (Oxoid, UK), stored at $4^{\circ} \mathrm{C}$, and sent to the microbiology laboratory within two hours. Samples were ground by tissue grinder in $0.5 \mathrm{ml}$ sterile saline and the homogenate was inoculated onto a Dent's selective medium (Oxoid, UK), which consisted of columbia blood agar base, $7 \%$ laked horse blood, and mixtures of antimicrobial and antifungal agents (vancomycin $10 \mathrm{mg} / \mathrm{l}$, trimethoprin $5 \mathrm{mg} / \mathrm{l}$, cefsulodin $5 \mathrm{mg} / \mathrm{l}$, and amphotericin B $5 \mathrm{mg} / \mathrm{l}$ ). They were incubated for up to seven days at $37^{\circ} \mathrm{C}$ under microaerophilic conditions $\left(80 \% \mathrm{O}_{2}, 15 \% \mathrm{~N}_{2}\right.$, and $5 \% \mathrm{CO}_{2}$ ) as described before. ${ }^{18} \mathrm{H}$ pylori colonies were confirmed by a positive urease, oxidase, and catalase test together with the identification of a Gram negative spiral looking organism on microscopy. Metronidazole susceptibility was determined by using the modified Kirby-Bauer disc diffusion procedure as described by DeCross et al. ${ }^{18}$ Briefly, $H$ pylori colonies were harvested into brain heart infusion broth (Oxoid, UK) plus 10\% horse serum to produce a cloudy suspension of McFarland no 3 to 4 . A sterile swab was dipped into the $H$ pylori suspension, squeezed out, and streaked evenly across a 5\% horse blood agar plate. Duplicate plates were made on each isolate. They were briefly dried, and then a $6 \mathrm{~mm}$ disc containing $5 \mu \mathrm{g} / \mathrm{ml}$ metronidazole was added to each plate before they were subjected to microaerophilic incubation as described above for seven days. The inhibition zone diameters were recorded then. An inhibition zone diameter of $>15 \mathrm{~mm}$ was regarded as metronidazole susceptible, ${ }^{18}$ and resistant to metronidazole if it was less than $15 \mathrm{~mm}$.

\section{Statistical analysis}

The $x^{2}$ and Fisher exact tests were used to compare the difference in percentage of metronidazole resistant $H$ pylori strains between the peptic ulcer disease group and the healthy volunteers. Furthermore, they were also used to test the difference in the eradication rates between the metronidazole susceptible and the metronidazole resistant $H$ pylori strains. The Mann-Whitney U test was used to show if there was any age difference between men and women within the peptic ulcer and the control groups. A p value of $<0.05$ was considered significant.

\section{Results}

$\mathrm{H}$ pylori culture rate and the prevalence of metronidazole resistance (Table)

A total of 80 (44 peptic ulcer and 25 healthy) subjects were found to be $H$ pylori positive by a combination of urea broth test and histology. Sixty nine $(86 \%)$ were successfully cultured by the techniques described above. Metronidazole resistance was found in 37 of $69(53.5 \%)$ and metronidazole susceptible in 32 of $69(46.5 \%)$ of the primary isolates (Table). Among the peptic ulcer disease group, 17 of $44(38.6 \%)$ were found to be metronidazole resistant. However, in the healthy subjects, 20 of $25(80 \%)$ were metronidazole resistant. There was significantly higher metronidazole resistant $H$ pylori strains present in the healthy than in the peptic ulcer disease group $(p=0 \cdot 0007)$. However, no significant difference in the metronidazole resistant $H$ pylori strains was seen between the men and the women in either the peptic ulcer disease group $(p=0.052)$ or the healthy subjects $(p=0 \cdot 19)$. But the overall prevalence of metronidazole resistant $H$ pylori strains was significantly higher in women ( 23 of $32,73 \%$ ) than in men (14 of $37,38 \%)(p=0.005)$. There was no significant age difference between those carrying the metronidazole resistant strains and those carrying the metronidazole susceptible ones $(p=0.093)$. However, women (mean age $58 \cdot 1$ years) were significantly older than men (mean age 45.8 years) in those with a positive $H$ pylori culture $(\mathrm{p}=0.008)$. In the culture negative group, there was no age difference between the men (mean age 48.3 years) and the women (mean age 57 years) $(p=0.082)$. Similarly, there was no age difference between the men and the women both among the peptic ulcer disease and the

Metronidazole susceptibility of the 69 Helicobacter pylori isolates

\begin{tabular}{lcc}
\hline & $\begin{array}{l}\text { Peptic ulcer disease } \\
(n=44)\end{array}$ & $\begin{array}{c}\text { Controls } \\
(n=25)\end{array}$ \\
\hline Metronidazole resistant & & \\
$\quad$ Males & 8 & 6 \\
Females & 9 & 14 \\
$\quad$ Total & 17 & 20 \\
Metronidazole sensitive & & \\
$\quad$ Males & 20 & 3 \\
Females & 7 & 2 \\
Total & 27 & 5 \\
\hline
\end{tabular}


healthy volunteer groups in the $H$ pylori culture positive subjects.

\section{Differential $\mathrm{H}$ pylori eradication rates between metronidazole susceptible and the metronidazole resistant groups}

Nineteen of 25 duodenal ulcer patients who received triple therapy were found to be negative for $H$ pylori infection. There was a significantly higher $H$ pylori eradication rate in those with metronidazole susceptible strains (14 of $15 ; 93 \%$ ) than the metronidazole resistant strains (five of $10 ; 50 \%)(p=0.021)$. For metronidazole resistant strains, there was no difference in the eradication rate between the amoxycillin (two of five) and the clarithromycin (three of five) containing anti- $H$ pylori regimens.

\section{Discussion}

Metronidazole resistant $H$ pylori strains occurred in about half of the culture positive subjects in this study. However, the prevalence of metronidazole resistant $H$ pylori was disproportionately higher in the healthy subjects than in the peptic ulcer disease patients, being roughly twice as frequent in the former. The prevalence of metronidazole resistant $H$ pylori strains in Hong Kong is somewhere between that of the developed and the developing countries. ${ }^{13-15}$ Interestingly, those carrying the metronidazole resistant strain were not significantly older than those carrying the metronidazole susceptible ones. Although it has been noticed in previous studies that there is increasing $H$ pylori infection carrier rates with age, age however in itself may not be a determining factor in metronidazole resistance property as borne out by this study. On the other hand, a significantly higher rate of metronidazole resistant $H$ pylori among the women, whether in this study or the others, ${ }^{8-13}$ could be perhaps accounted for by the previous history of nitroimidazole ingestion for pelvic inflammatory diseases. Unlike the developing countries such as Africa, metronidazole is not so widely used in Hong Kong because there is a much lower rate of giardiasis or amoebiasis. As a result, there is a much lower prevalence of metronidazole resistant $H$ pylori strains in Hong Kong. The fact that there is a significantly lower metronidazole resistant $H$ pylori rate among the peptic ulcer disease patients compared with the healthy subjects is encouraging for clinicians. This is supported by the high $H$ pylori eradication rates (about $80 \%$ ) achieved by using combination therapies that consist of metronidazole as one of the antimicrobial agents $^{219}$ among the Chinese in Hong Kong. It has been reported in a number of previous studies that the metronidazole resistant $H$ pylor $i$ strain is much more difficult to eradicate. ${ }^{8-12}$ This is further supported by our study. The choice of combination therapy may have to be tailored according to the susceptibility of the organism towards metronidazole. ${ }^{20-23}$

It has been suggested by some that routine culture and sensitivity against metronidazole should be performed in cases where anti- $H$ pylori therapy is contemplated so that a much higher eradication rate could be achieved by using the appropriate combination therapy. ${ }^{20}$ This approach may not be as practical as it seems because it is quite a tedious and time consuming process. On average, it takes about 10 working days to confirm the susceptibility of the organism against metronidazole. This approach will undoubtedly confer delay in initiating therapy for those requiring treatment. The added problem to this is the false negative culture result, which occurs between $10-20 \%$. We would suggest that triple therapy consisting of either a bismuth compound or omeprazole in combination with amoxycillin and metronidazole be given to ulcer patients while waiting for metronidazole resistance results. This combination therapy will (a) heal the ulcer effectively, and (b) satisfy the patients who require active therapy without any delay. This combination, albeit with a lower eradication rate in those with metronidazole resistant strains, would not induce further antibiotic resistance in the organism because no amoxycillin resistant $H$ pylori strains have been reported yet. If subsequent results show that the organism is indeed metronidazole resistant and there is a failure of eradication by confirmatory testing at least four weeks after completion of the antibiotics, alternative combination therapy such as reported by Logan et al ${ }^{20}$ could be tried on another occasion. This approach may be the best compromise until an ideal combination therapy has been found that can eradicate $H$ pylori effectively irrespective of metronidazole susceptibility.

1 Marshall BJ. Helicobacter pylori. Am $\mathcal{f}$ Gastroenterol 1994 89 (suppl): S116-28.

2 Hosking SW, Ling TKW, Chung SCS, Yung MY, Cheng $\mathrm{AF}$, Sung JJ, et al. Duodenal ulcer healing by eradication of Helicobacter pylori without anti-acid treatment: randomised controlled trial. Lancet 1994; 343: 508-10.

3 Thijs JC, Zwet AA, van Oey HB. Efficacy and side effects of a triple drug regimen for the eradication of Helicobacter pylori. Scand $\mathcal{F}$ Gastroenterol 1994; 28: 934-8.

4 Graham DY, Lew GM, Klein PD, Evans DG, Evans DJ Jr, Saeed ZA, et al. Effect of treatment of Helicobacter pylori infection on the long-term recurrence of gastric or duodenal ulcer: a randomized controlled study. Ann Intern Med 1992; 116: 705-8.

5 Labenz J, Borsch G. Evidence for the essential role of Helicobacter pylori in gastric ulcer disease. Gut 1994; 35: 19-22.

6 Sung JJY, Chung SCS, Ling TKW, Yung MY, Leung VKS, $\mathrm{Ng}$ EKW, et al. Antibacterial treatment of gastric ulcers associated with Helicobacter pylori. $N$ Engl f Med 1995; 332: 139-42.

$7 \mathrm{NIH}$ Consensus. Helicobacter pylori in peptic ulcer disease. ЭAMA 1994; 272: 65-9.

8 Rautelin H, Seppala K, Renkonen O, Vainio U, Kosunen TU. Role of metronidazole resistance in therapy of Helicobacter pylori infections. Antimicrob Agents Chemother 1992; 36: 163-6.

9 Bell GD, Powell K, Burridge A, Pallecaros A, Jones PH, Gant PW, et al. Experience with triple anti-Helicobacter pylori eradication therapy: side effects and the importance of testing the pre-treatment bacterial isolate for metronidazole resistance. Aliment Pharmacol Ther 1992; 6: 427-35.

10 Logan RPH, Gummett PA, Misiewicz J, Karim QN, Walker $\mathrm{MM}$, Baron JH. One week eradication regimen for Helicobacter pylori. Lancet 1991; 338: 1249-52.

11 Graham DY, Lew GM, Malaty HM, Evans DG, Evans DJ $\mathrm{Jr}$, Klein PD, et al. Factors influencing the eradication of Helicobacter pylori with triple therapy. Gastroenterology 1992; 102: 493-6.

12 Thijs JC, Zwet AA, van Oey HB. Efficacy and side effects of a triple drug regimen for the eradication of Helicobacter pylori. Scand $\mathcal{f}$ Gastroenterol 1993; 28: 934-8.

13 Glupczynski Y, Burette A, Goossens H, DePrez C, Butzler JP. Results of a multicentre European Survey in 1991 of metronidazole resistance in Helicobacter pylori. Eur f Clin
Microbiol Infect Dis 1992; 11: 777-81. 
14 Glupcznski Y, Burrette A, DeKoster E, Nust JF, Deltenre $M$, Cadranel S, et al. Metronidazole resistance in Helicobacter pylori. Lancet 1990; 335: 976-7.

15 Al-Assi MT, Ramirez FC, Lew GM, Genta RM, Graham DY. Clarithromycin, tetracycline, and bismuth: a new DY. Clarithromycin, tetracycline, and bismuth: non-metronidazole therapy for Helicobacter pylori infection. Am F Gastroenterol 1994; 89: 1203-5.

6 Ching CK, Buxton C, Holgate C, Holmes GKT. Cytological brushing urea broth test - a highly sensitive and specific test for Helicobacter pylori infection. Gastrointest Endosc 1991; 37: 550-1.

17 Hui WM, Lam SK, Chau PY, Ho J, Lau WY, Poon KP, et al. Pathogenetic role of Campylobacter in gastric ulcer. 7 Gastroenterol Hepatol 1987; 2: 309-16.

18 DeCross AJ, Marshall BJ, McCallum RW, Hoffman SR, Barrett LJ, Guerrant RL. Metronidazole susceptibility testing for Helicobacter pylori: comparison of disk, broth, and agar dilution methods and their clinical relevance. f Clin Microbiol 1993; 31: 1971-4.
19 Lam SK, Hu WHC, Ching CK. Sucralfate in Helicobacter pylori eradication strategies. Scand $\mathcal{f}$ Gastroenterol (in press)

20 Logan RPH, Gummett PA, Misiewicz J, Karim QN, Walker MM, Baron JH. Two-week eradication regimen
for metronidazole-resistant Helicobacter pylori. Aliment for metronidazole-resistant Helicob

21 Glupczynski Y, Buordeaux L, Verhas M. Short-term double or triple oral drug treatment of Helicobacter pylori $(\mathrm{Hp})$ in Central Africa. Gastroenterology 1990; 98: A48.

22 Bell GD, Powell KU, Burridge SM, Spencer G, Bolton G, Purser K, et al. Short report: Omeprazole plus antibiotic combinations for the eradication of metronidazole-resistant Helicobacter pylori. Aliment Pharmacol Ther 1992; 6 : 751-8.

23 Thijs JC, Zwet AA, van Moolenaar W, Oom JAJ, DeKores $\mathrm{H}$, Runhaar A Clarithromycin, an alternative to metronidazole in the triple therapy of Helicobacter pylori infecdazole in the triple therapy of Helicobacter
tion. Aliment Pharmacol Ther $1994 ; 8: 131-4$. 